\title{
Dialyzable Carbosilane Dendrimers as Soluble Supports for the Functionalization of Pyridine Fragments via Palladium-Catalyzed
} Coupling Reactions.

\author{
Jérôme Le Nôtre ${ }^{\mathrm{a}}$, Judith J. Firet ${ }^{\mathrm{a}}$, Leo A. J. M. Sliedregt ${ }^{\mathrm{b}}$, Bart J. van Steen ${ }^{\mathrm{b}}$, \\ Gerard van Koten ${ }^{\mathrm{a}}$ and Robertus J. M. Klein Gebbink ${ }^{\mathrm{a}, *}$ \\ ${ }^{a}$ Debye Institute, Department of Metal-Mediated Synthesis and Homogeneous \\ Catalysis, Utrecht University, Padualaan 8, 3584 CH Utrecht, The Netherlands. \\ ${ }^{b}$ Solvay Pharmaceuticals B.V., Lead Discovery Unit, C.J. van Houtenlaan 36, 1381 \\ CP Weesp, The Netherlands. \\ r.j.m.kleingebbink@chem.uu.nl
}

\section{Experimental data}

General: All syntheses were performed under an atmosphere of dry nitrogen using standard Schlenk techniques. Solvents were dried and distilled prior to use. ${ }^{1} \mathrm{H}$, ${ }^{13} \mathrm{C}\left\{{ }^{1} \mathrm{H}\right\}$ and ${ }^{29} \mathrm{Si}$ NMR spectra were recorded using a Varian Inova $300 \mathrm{MHz}$ spectrometer. Microanalyses were performed by Kolbe Microanalytical Laboratories, Mühlheim a/d Ruhr, Germany. Membranes used for passive dialysis are commercially available (Sigma: Dialysis Tubing, benzoylated $\left(\right.$ cut off $\left.=1200 \mathrm{~g} \cdot \mathrm{mol}^{-1}\right)$.

General procedure for the linkage reaction:(i) To a solution of dry diisopropylamine (2.2 equiv) in THF (1.2 $\mathrm{mL}$ per mmol of 3-bromopyridine) under argon at $-80{ }^{\circ} \mathrm{C}$ was added via syringe $n$-butyllithium (1.6 $\mathrm{M}$ in hexane, 2.2 equiv). This solution was stirred at $-80{ }^{\circ} \mathrm{C}$ for 30 minutes, cooled down to $-105{ }^{\circ} \mathrm{C}$ and then was treated via syringe by a slow addition of 3-bromopyridine (2 equiv), keeping internal temperature below $-100{ }^{\circ} \mathrm{C}$. The reaction mixture was stirred 10 minutes at 
$-100{ }^{\circ} \mathrm{C}$ and the resulting lithiopyridine complex was quenched with a solution of the electrophile (1 equiv) in THF ( $2 \mathrm{~mL}$ per mmol of electrophile). After a slow warm up from $-100{ }^{\circ} \mathrm{C}$ to $0{ }^{\circ} \mathrm{C}$ (ca. $2 \mathrm{~h}$ ), the reaction mixture was hydrolysed by addition of triethylamine (2.2 equiv) and methanol (2.2 equiv). Then the mixture was allowed to room temperature and extracted two times with ethyl acetate and water. The combined organic layers were washed with brine, dried over $\mathrm{MgSO}_{4}$, filtered and evaporated to dryness. The crude was purified by column chromatography using silica gel and ethyl acetate as eluent or by passive dialysis to give the corresponding linked pyridine.

3-bromo-4-trimethylsilanylpyridine 2: (ii) Diisopropylamine (4.1 mL, $29.2 \mathrm{mmol})$, nbutyllithium (18.2 mL, $29.2 \mathrm{mmol})$, THF (32 mL), then 3-bromopyridine 1 (2.57 mL, $26.7 \mathrm{mmol})$, chlorotrimethylsilane $(1.5 \mathrm{~mL}, 12.2 \mathrm{mmol})$ in $10 \mathrm{~mL}$ of THF. Column chromatography using pure ethyl acetate afforded the 3-bromo-4trimethylsilanylpyridine 2 as colorless oil $(2.56 \mathrm{~g}, 91 \%$ yield $) .{ }^{1} \mathrm{H}$ NMR $\left(\mathrm{CDCl}_{3}\right): \delta$ $8.58(\mathrm{~s}, 1 \mathrm{H}, \mathrm{Ar}-\mathrm{H}), 8.40\left(\mathrm{~d}, 1 \mathrm{H},{ }^{3} \mathrm{~J}_{\mathrm{H}-\mathrm{H}}=4.8 \mathrm{~Hz}, \mathrm{Ar}-\mathrm{H}\right), 7.24\left(\mathrm{~d}, 1 \mathrm{H},{ }^{3} \mathrm{~J}_{\mathrm{H}-\mathrm{H}}=4.8 \mathrm{~Hz}\right.$, Ar-H), $0.35\left(\mathrm{~s}, 9 \mathrm{H}, \mathrm{Si}-\mathrm{CH}_{3}\right) .{ }^{13} \mathrm{C} \mathrm{NMR}\left(\mathrm{CDCl}_{3}\right): \delta 150.4(\mathrm{Ar}-\mathrm{CH}), 149.5$ (quaternary C), 146.2, $129.3(2 \times \mathrm{Ar}-\mathrm{CH}), 127.8$ (quaternary $\mathrm{C}$ ), $-2.1\left(\mathrm{Si}\left(\mathrm{CH}_{3}\right)_{2}\right) .{ }^{29} \mathrm{Si} \mathrm{NMR}$ $\left(\mathrm{CDCl}_{3}\right): \delta 0.22$.

[3-bromo-4-G0-pyridine] 11: Diisopropylamine (0.68 mL, $4.84 \mathrm{mmol}), \mathrm{n}$ butyllithium (3.0 mL, $4.84 \mathrm{mmol})$, THF (7 mL), then 3-bromopyridine $1(0.43 \mathrm{~mL}$, $4.46 \mathrm{mmol})$, chlorocarbosilane-G0 9 (0.43 g, $0.75 \mathrm{mmol})$ in $5 \mathrm{~mL}$ of THF. Column chromatography using pure ethyl acetate afforded the 3-bromo-4-G0-pyridine $\mathbf{1 1}$ as colorless oil $\left(0.48 \mathrm{~g}, 61 \%\right.$ yield). ${ }^{1} \mathrm{H}$ NMR $\left(\mathrm{CDCl}_{3}\right): \delta 8.58(\mathrm{~s}, 4 \mathrm{H}, \mathrm{Ar}-\mathrm{H}), 8.40(\mathrm{~d}, 4$ $\left.\mathrm{H},{ }^{3} \mathrm{~J}_{\mathrm{H}-\mathrm{H}}=4.5 \mathrm{~Hz}, \mathrm{Ar}-\mathrm{H}\right), 7.22\left(\mathrm{~d}, 4 \mathrm{H},{ }^{3} \mathrm{~J}_{\mathrm{H}-\mathrm{H}}=4.5 \mathrm{~Hz}, \mathrm{Ar}-\mathrm{H}\right), 1.24-1.15(\mathrm{~m}, 8 \mathrm{H}, \mathrm{C}-$ $\mathrm{CH}_{2}-\mathrm{C}$ ), 0.98-0.94 (m, 8 H, CH $\left.2-\mathrm{Si}-\mathrm{Ar}\right), 0.48-0.40$ (m, 8 H, $\left.\mathrm{CH}_{2}-\mathrm{Si}_{-}-\mathrm{CH}_{2}\right), 0.32$ (s, 24 
$\left.\mathrm{H}, \mathrm{Si}-\mathrm{CH}_{3}\right) .{ }^{13} \mathrm{C} \mathrm{NMR}\left(\mathrm{CDCl}_{3}\right): \delta 151.0(\mathrm{Ar}-\mathrm{CH}), 149.9$ (quaternary C), 146.7, 130.2 (2 x Ar-CH), 128.4 (quaternary C), 19.1, 18.2, $16.9\left(3 \times \mathrm{CH}_{2}\right),-3.0\left(\mathrm{Si}\left(\mathrm{CH}_{3}\right)_{2}\right) .{ }^{29} \mathrm{Si}$ NMR $\left(\mathrm{CDCl}_{3}\right): \delta 0.78$ (Si core), $0.66(4 \mathrm{x} \mathrm{Si})$. Anal. Calc. for $\mathrm{C}_{40} \mathrm{H}_{60} \mathrm{Br}_{4} \mathrm{~N}_{4} \mathrm{Si}_{5}: \mathrm{C}$ 45.45, H 5.72, N 5.30, Si 13.29. Found: C 45.58, H 5.70, N 5.21, Si 13.32.

[3-bromo-4-G1-pyridine]: G1-(3BrPy $)_{10}\left(\mathrm{OCH}_{3}\right)_{2}$ 12: Diisopropylamine $(2.1 \mathrm{~mL}$, $14.7 \mathrm{mmol})$, n-butyllithium $(9.2 \mathrm{~mL}, 14.7 \mathrm{mmol})$, THF $(16 \mathrm{~mL})$, then 3 bromopyridine 1 (1.3 mL, $13.4 \mathrm{mmol})$, chlorocarbosilane-G1 10 (1.08 g, $0.56 \mathrm{mmol})$ in $10 \mathrm{~mL}$ of THF. Hydrolysis with $\mathrm{NEt}_{3}(2.1 \mathrm{~mL}, 14.7 \mathrm{mmol})$ and methanol $(0.6 \mathrm{~mL}$, $14.7 \mathrm{mmol}$ ). The crude material (ca. $1.5 \mathrm{~g}$ ) was diluted in $20 \mathrm{~mL}$ of technical methanol and placed into a membrane bag. This bag was then placed into a beaker containing $400 \mathrm{~mL}$ of methanol and the solution was slowly stirred overnight at room temperature. Evaporation of the internal solution of the bag afforded the G1$(3 \mathrm{BrPy})_{10}\left(\mathrm{OCH}_{3}\right)_{2} 12$ as brown oil $(1.30 \mathrm{~g}, 74 \%$ yield $) .{ }^{1} \mathrm{H}$ NMR $\left(\mathrm{CDCl}_{3}\right): \delta 8.53$ (br s, $10 \mathrm{H}, \mathrm{Ar}-\mathrm{H}), 8.36$ (br s, $10 \mathrm{H}, \mathrm{Ar}-\mathrm{H}), 7.18$ (br s, $10 \mathrm{H}, \mathrm{Ar}-\mathrm{H}), 3.30$ (s, $6 \mathrm{H}, 2 \mathrm{x}$ $\mathrm{OCH}_{3}$ ), 1.40-1.08 (m, $40 \mathrm{H}, \mathrm{C}-\mathrm{CH}_{2}-\mathrm{C}$ ), 0.89 (br s, $24 \mathrm{H}, \mathrm{CH}_{2}$-Si-Ar), 0.44 (br s, $32 \mathrm{H}$, $\left.\mathrm{CH}_{2}-\mathrm{Si}-\mathrm{CH}_{2}\right), 0.27$ (s, $\left.60 \mathrm{H}, \mathrm{Si}\left(\mathrm{CH}_{3}\right)_{2}(\mathrm{Ar})\right),-0.01$ (s, $\left.12 \mathrm{H}, \mathrm{Si}\left(\mathrm{CH}_{3}\right)_{2}\left(\mathrm{OCH}_{3}\right)\right) .{ }^{13} \mathrm{C}$ $\operatorname{NMR}\left(\mathrm{CDCl}_{3}\right): \delta 151.0(\mathrm{Ar}-\mathrm{CH}), 149.8$ (quaternary C), 146.7, $130.2(2 \mathrm{x} \mathrm{Ar}-\mathrm{CH})$, 128.4 (quaternary C), $47.3\left(\mathrm{OCH}_{3}\right), 20.9,20.6,19.9,19.8,19.1,18.3,17.7,17.0$ $\left(\mathrm{CH}_{2}\right),-2.6\left(\mathrm{Si}\left(\mathrm{CH}_{3}\right)_{2}(\mathrm{Ar})\right),-3.0\left(\mathrm{Si}\left(\mathrm{CH}_{3}\right)_{2}\left(\mathrm{OCH}_{3}\right)\right) .{ }^{29} \mathrm{Si} \mathrm{NMR}\left(\mathrm{CDCl}_{3}\right): \delta 18.7(2 \mathrm{x}$ Si-OCH 3 ), 0.79 (Si core), 0.61 (10 x Si-Ar), -3.91 (Si inner).

G1-(3BrPy) 12 13: Diisopropylamine (1.67 mL, $11.9 \mathrm{mmol})$, n-butyllithium (7.4 mL, $11.9 \mathrm{mmol})$, THF $(15 \mathrm{~mL})$, then 3-bromopyridine $1(1.04 \mathrm{~mL}, 10.8 \mathrm{mmol})$, chlorocarbosilane-G1 10 (0.87 g, $0.45 \mathrm{mmol})$ in $10 \mathrm{~mL}$ of THF. Hydrolysis with $\mathrm{NEt}_{3}$ (1.7 mL, $11.9 \mathrm{mmol})$ and methanol $(0.5 \mathrm{~mL}, 11.9 \mathrm{mmol})$. The crude material (ca. 1.5 g) was diluted in $20 \mathrm{~mL}$ of technical methanol and placed into a membrane bag. This 
bag was then placed into a beaker containing $400 \mathrm{~mL}$ of methanol and the solution was slowly stirred overnight at room temperature. Evaporation of the internal solution of the bag afforded the G1-(3BrPy $)_{12} 13$ as brown oil $\left(1.17 \mathrm{~g}, 77 \%\right.$ yield). ${ }^{1} \mathrm{H}$ NMR $\left(\mathrm{CDCl}_{3}\right): \delta 8.51$ (br s, $\left.12 \mathrm{H}, \mathrm{Ar}-\mathrm{H}\right), 8.33$ (br s, $\left.12 \mathrm{H}, \mathrm{Ar}-\mathrm{H}\right), 7.17$ (br s, $12 \mathrm{H}, \mathrm{Ar}-\mathrm{H}$ ), 1.44-1.01 (m, $40 \mathrm{H}, \mathrm{C}-\mathrm{CH}_{2}-\mathrm{C}$ ), 0.90 (br s, $24 \mathrm{H}, \mathrm{CH}_{2}-\mathrm{Si}-\mathrm{Ar}$ ), 0.41 (br s, $32 \mathrm{H}, \mathrm{CH}_{2}-$ $\left.\mathrm{Si}-\mathrm{CH}_{2}\right), 0.24\left(\mathrm{~s}, 72 \mathrm{H}, \mathrm{Si}\left(\mathrm{CH}_{3}\right)_{2}(\mathrm{Ar})\right) .{ }^{13} \mathrm{C} \mathrm{NMR}\left(\mathrm{CDCl}_{3}\right): \delta 150.9(\mathrm{Ar}-\mathrm{CH}), 149.6$ (quaternary C), 146.6, 130.1 (2 x Ar-CH), 128.4 (quaternary C), 21.0, 20.1, 19.1, 18.3, 17.6, $17.0\left(\mathrm{CH}_{2}\right),-3.0\left(\mathrm{Si}\left(\mathrm{CH}_{3}\right)_{2}(\mathrm{Ar})\right) .{ }^{29} \mathrm{Si} \mathrm{NMR}\left(\mathrm{CDCl}_{3}\right): \delta 0.77$ (Si core), 0.52 (10 x Si-Ar), -4.02 (Si inner).

General procedure for the Suzuki coupling reaction: ${ }^{(i i i)}$ To a solution of silylated pyridine ( 1 equiv) in degassed toluene $(5 \mathrm{~mL}$ per mmol) and degassed EtOH $(1 \mathrm{~mL}$ per mmol) were added $\mathrm{Pd}\left(\mathrm{PPh}_{3}\right)_{4}(1$ or $2 \mathrm{~mol} \%), 2 \mathrm{M} \mathrm{Na}_{2} \mathrm{CO}_{3}$ degassed solution (2 $\mathrm{mL}, 5$ equiv) and the boronic acid (1.5 equiv). The mixture was vigorously stirred and heated at reflux for 16 hours. After cooling down and addition of water, the mixture was extracted two times with ethyl acetate. The combined organic layers were washed with aqueous $1 \mathrm{M} \mathrm{NaHCO}_{3}$ solution then water, dried over $\mathrm{MgSO}_{4}$, filtered and evaporated to dryness. Column chromatography over silica gel using an appropriate mixture of ethyl acetate and hexane or passive dialysis afforded the corresponding biaryl compounds.

3-p-tolyl-4-trimethylsilanylpyridine 3: 3-bromo-4-trimethylsilanylpyridine 2 (0.46 g, $2.0 \mathrm{mmol})$ in $10 \mathrm{~mL}$ of degassed toluene and $2 \mathrm{~mL}$ of degassed ethanol, $\mathrm{Pd}\left(\mathrm{PPh}_{3}\right)_{4}$ (23.1 mg, $1 \mathrm{~mol} \%), \mathrm{Na}_{2} \mathrm{CO}_{3}(5 \mathrm{~mL}, 2 \mathrm{M}$ degassed solution), $p$-tolueneboronic acid (0.408 g, $3.0 \mathrm{mmol})$. Column chromatography using ethyl acetate/hexane (1/1) afforded the 3-p-tolyl-4-trimethylsilanylpyridine 3 as colorless oil $(0.411 \mathrm{~g}, 85 \%$ 
yield). ${ }^{1} \mathrm{H}$ NMR $\left(\mathrm{CDCl}_{3}\right): \delta 8.53\left(\mathrm{~d}, 1 \mathrm{H},{ }^{3} \mathrm{~J}_{\mathrm{H}-\mathrm{H}}=4.8 \mathrm{~Hz}, \mathrm{Ar}-\mathrm{H}\right), 8.43(\mathrm{~s}, 1 \mathrm{H}, \mathrm{Ar}-\mathrm{H})$, $7.44\left(\mathrm{~d}, 1 \mathrm{H},{ }^{3} \mathrm{~J}_{\mathrm{H}-\mathrm{H}}=4.8 \mathrm{~Hz}, \mathrm{Ar}-\mathrm{H}\right), 7.21\left(\mathrm{~d}(\mathrm{AB}), 2 \mathrm{H},{ }^{3} \mathrm{~J}_{\mathrm{H}-\mathrm{H}}=7.8 \mathrm{~Hz}\right.$, Ar-H Phenyl), $7.16\left(\mathrm{~d}(\mathrm{AB}), 2 \mathrm{H},{ }^{3} \mathrm{~J}_{\mathrm{H}-\mathrm{H}}=7.8 \mathrm{~Hz}\right.$, Ar-H Phenyl), 2.40 (s, $\left.3 \mathrm{H}, \mathrm{Ar}-\mathrm{CH}_{3}\right), 0.04$ (s, $9 \mathrm{H}$, $\left.\mathrm{Si}-\mathrm{CH}_{3}\right) .{ }^{13} \mathrm{C}$ NMR $\left(\mathrm{CDCl}_{3}\right): \delta 149.3(\mathrm{Ar}-\mathrm{CH}), 148.0$ (quaternary C), $147.0(\mathrm{Ar}-\mathrm{CH})$, 143.9, 137.8, 137.4 (3 x quaternary C), 129.3, 128.6, 128.5 (3 x Ar-CH), $21.2(\mathrm{Ar}-$ $\left.\mathrm{CH}_{3}\right),-0.2\left(\mathrm{Si}\left(\mathrm{CH}_{3}\right)_{2}\right) .{ }^{29} \mathrm{Si} \mathrm{NMR}\left(\mathrm{CDCl}_{3}\right): \delta$-2.78. Anal. Calc. for $\mathrm{C}_{15} \mathrm{H}_{19} \mathrm{NSi}: \mathrm{C}$ 74.63, H 7.93, N 5.80, Si 11.63. Found: C 74.71, H 7.86, N 5.74, Si 11.58.

[3-p-tolyl-4-G0-pyridine] 14: 3-bromo-4-G0-pyridine 11 (400 mg, $0.38 \mathrm{mmol}$ ) in 10 $\mathrm{mL}$ of degassed toluene and $2 \mathrm{~mL}$ of degassed ethanol, $\mathrm{Pd}\left(\mathrm{PPh}_{3}\right)_{4}(17.5 \mathrm{mg}, 4 \mathrm{x} 1$ mol\%), $\mathrm{Na}_{2} \mathrm{CO}_{3}$ (3.8 mL, $2 \mathrm{M}$ degassed solution), $p$-tolueneboronic acid (309 mg, $2.27 \mathrm{mmol})$. Column chromatography using ethyl acetate/hexane (1/1) afforded the 3p-tolyl-4-G0-pyridine 14 as colorless oil (380 mg, 91\% yield). ${ }^{1} \mathrm{H}$ NMR $\left(\mathrm{CDCl}_{3}\right): \delta$ $8.49\left(\mathrm{~d}, 4 \mathrm{H},{ }^{3} \mathrm{~J}_{\mathrm{H}-\mathrm{H}}=4.8 \mathrm{~Hz}, \operatorname{Ar}-\mathrm{H}\right), 8.40(\mathrm{~s}, 4 \mathrm{H}, \mathrm{Ar}-\mathrm{H}), 7.37\left(\mathrm{~d}, 4 \mathrm{H},{ }^{3} \mathrm{~J}_{\mathrm{H}-\mathrm{H}}=4.8 \mathrm{~Hz}\right.$, Ar-CH), $7.17\left(\mathrm{~d}(\mathrm{AB}), 8 \mathrm{H},{ }^{3} \mathrm{~J}_{\mathrm{H}-\mathrm{H}}=7.8 \mathrm{~Hz}\right.$, Ar-H Phenyl), $7.11\left(\mathrm{~d}(\mathrm{AB}), 8 \mathrm{H},{ }^{3} \mathrm{~J}_{\mathrm{H}-\mathrm{H}}=\right.$ 7.8 Hz, Ar-H Phenyl), 2.38 (s, 12 H, Ar- $\mathrm{CH}_{3}$ ), 1.15-1.04 (m, $8 \mathrm{H}, \mathrm{C}-\mathrm{CH}_{2}-\mathrm{C}$ ), 0.520.45 (m, $\left.8 \mathrm{H}, \mathrm{CH}_{2}-\mathrm{Si}-\mathrm{Ar}\right), 0.36-0.25\left(\mathrm{~m}, 8 \mathrm{H}, \mathrm{CH}_{2}-\mathrm{Si}_{-}-\mathrm{CH}_{2}\right),-0.05$ (s, $\left.24 \mathrm{H}, \mathrm{Si}-\mathrm{CH}_{3}\right)$. ${ }^{13} \mathrm{C} \mathrm{NMR}\left(\mathrm{CDCl}_{3}\right): \delta 149.3(\mathrm{Ar}-\mathrm{CH}), 147.3$ (quaternary C), $146.9(\mathrm{Ar}-\mathrm{CH}), 144.0$, 137.7, 137.4, 132.1 (quaternary C), 129.3, 128.7, 128.6, $128.4(\mathrm{Ar}-\mathrm{CH}), 21.2(\mathrm{Ar}-$ $\left.\mathrm{CH}_{3}\right), 20.9,18.4,17.2\left(3 \times \mathrm{CH}_{2}\right),-1.9\left(\mathrm{Si}\left(\mathrm{CH}_{3}\right)_{2}\right) .{ }^{29} \mathrm{Si} \mathrm{NMR}\left(\mathrm{CDCl}_{3}\right): \delta 0.38(\mathrm{Si}$ core$)$, -2.45 (4 x Si). Anal. Calc. for $\mathrm{C}_{68} \mathrm{H}_{88} \mathrm{~N}_{4} \mathrm{Si}_{5}$ : C 74.12, H 8.05, N 5.08, Si 12.74. Found: C 74.21, H 8.11, N 4.97, Si 12.61.

[3-p-tolyl-4-G1-pyridine]: G1-(3-p-tolylPy) ${ }_{12}$ 15: G1-(3BrPy) $)_{12} 13$ (200 mg, 0.06 mmol) in $10 \mathrm{~mL}$ of degassed toluene and $2 \mathrm{~mL}$ of degassed ethanol, $\mathrm{Pd}\left(\mathrm{PPh}_{3}\right)_{4}(16.3$ mg, 12x1 mol\%), $\mathrm{Na}_{2} \mathrm{CO}_{3}$ (1.8 mL, $2 \mathrm{M}$ degassed solution), $p$-tolueneboronic acid (144 mg, $1.06 \mathrm{mmol}$ ). The crude material (ca. $0.4 \mathrm{~g}$ ) was diluted in $10 \mathrm{~mL}$ of technical 
methanol and placed into a membrane bag. This bag was then placed into a beaker containing $200 \mathrm{~mL}$ of methanol and the solution was slowly stirred overnight at room temperature. Evaporation of the internal solution of the bag afforded the G1-(3-ptolylPy $)_{12} 15$ as brown oil $\left(160 \mathrm{mg}, 77 \%\right.$ yield). ${ }^{1} \mathrm{H}$ NMR $\left(\mathrm{CDCl}_{3}\right): \delta 8.44$ (br s, $12 \mathrm{H}$, Ar-H), 8.38 (br s, 12 H, Ar-H), 7.32 (br s, 12 H, Ar-H), 7.25-7.05 (br m, 48 H, Ar-H Phenyl), 2.53 (s, $36 \mathrm{H}, \mathrm{Ar}-\mathrm{CH}_{3}$ ), 1.20-1.00 (m, $40 \mathrm{H}, \mathrm{C}-\mathrm{CH}_{2}-\mathrm{C}$ ), 0.65-0.25 (br m, 56 $\mathrm{H}, \mathrm{CH}_{2}-\mathrm{Si}-\mathrm{Ar}$ and $\left.\mathrm{CH}_{2}-\mathrm{Si}-\mathrm{CH}_{2}\right),-0.10\left(\mathrm{~s}, 72 \mathrm{H}, \mathrm{Si}\left(\mathrm{CH}_{3}\right)_{2}(\mathrm{Ar})\right) .{ }^{13} \mathrm{C} \mathrm{NMR}\left(\mathrm{CDCl}_{3}\right): \delta$ 149.3 (Ar-CH), 146.9 (quaternary C), 143.9, 137.6, 137.3 (quaternary C), 130.1, $128.4(2 \times \mathrm{Ar}-\mathrm{CH}), 21.1,21.0,20.1,18.4,17.4,17.2\left(\mathrm{CH}_{2}\right),-1.9\left(\mathrm{Si}\left(\mathrm{CH}_{3}\right)_{2}(\mathrm{Ar})\right) .{ }^{29} \mathrm{Si}$ NMR $\left(\mathrm{CDCl}_{3}\right): \delta 0.8$ (Si core), -2.5 (Si-Ar), -3.9 (Si inner).

General procedure for the Heck coupling reaction: (iv) To a solution of silylated pyridine ( 1 equiv) in dry $N, N$-dimethylformamide $(5 \mathrm{ml}$ per mmol) were added $\mathrm{Pd}(\mathrm{OAc})_{2}(2.5 \mathrm{~mol} \%), \mathrm{PPh}_{3}(5 \mathrm{~mol} \%), \mathrm{NEt}_{3}$ (5 equiv) and ethyl acrylate (1.5 equiv). The reaction mixture was heated 16 hours at $130{ }^{\circ} \mathrm{C}$. The mixture was then cooled down to room temperature, diluted with water, extracted with ethyl acetate and dried over $\mathrm{MgSO}_{4}$. The resulting solution was filtered through a short plug of Celite and evaporated to dryness. The crude material was then purified by column chromatography using an appropriate mixture of ethyl acetate and hexane or by passive dialysis.

3-(4-trimethylsilanylpyridin-3-yl)-acrylic acid ethyl ester 4: 3-bromo-4trimethylsilanylpyridine $2(0.50 \mathrm{~g}, 2.17 \mathrm{mmol})$ in $11 \mathrm{~mL}$ of DMF, $\mathrm{Pd}(\mathrm{OAc})_{2}(12.2$ $\mathrm{mg}, 2.5 \mathrm{~mol} \%), \mathrm{PPh}_{3}(28.5 \mathrm{mg}, 5 \mathrm{~mol} \%), \mathrm{NEt}_{3}(1.4 \mathrm{~mL}, 6.5 \mathrm{mmol})$ and ethyl acrylate (0.38 mL, $3.48 \mathrm{mmol})$. Column chromatography using ethyl acetate/hexane (1/2) afforded the 3-(4-trimethylsilanylpyridin-3-yl)-acrylic acid ethyl ester $\mathbf{4}$ as colorless 
oil (452 mg, 85\% yield). ${ }^{1} \mathrm{H}$ NMR $\left(\mathrm{CDCl}_{3}\right): \delta 8.71$ (br s, $\left.1 \mathrm{H}, \mathrm{Ar}-\mathrm{H}\right), 8.44\left(\mathrm{~d}, 1 \mathrm{H},{ }^{3} \mathrm{~J}_{\mathrm{H}-}\right.$ $\mathrm{H}=4.8 \mathrm{~Hz}, \mathrm{Ar}-\mathrm{H}), 7.91\left(\mathrm{~d}, 1 \mathrm{H},{ }^{3} \mathrm{~J}_{\mathrm{H}-\mathrm{H}}=15.8 \mathrm{~Hz}, \mathrm{CH}=\mathrm{CHCO}_{2} \mathrm{Et}\right), 7.33\left(\mathrm{~d}, 1 \mathrm{H},{ }^{3} \mathrm{~J}_{\mathrm{H}-\mathrm{H}}=\right.$ $4.8 \mathrm{~Hz}, \mathrm{Ar}-\mathrm{H}), 6.33\left(\mathrm{~d}, 1 \mathrm{H},{ }^{3} \mathrm{~J}_{\mathrm{H}-\mathrm{H}}=15.8 \mathrm{~Hz}, \mathrm{CH}=\mathrm{CHCO}_{2} \mathrm{Et}\right), 4.20\left(\mathrm{q}, 2 \mathrm{H},{ }^{3} \mathrm{~J}_{\mathrm{H}-\mathrm{H}}=7.1\right.$ $\left.\mathrm{Hz}, \mathrm{CH}_{2} \mathrm{CH}_{3}\right), 1.27\left(\mathrm{t}, 3 \mathrm{H},{ }^{3} \mathrm{~J}_{\mathrm{H}-\mathrm{H}}=7.1 \mathrm{~Hz}, \mathrm{CH}_{2} \mathrm{CH}_{3}\right), 0.31\left(\mathrm{~s}, 9 \mathrm{H}, \mathrm{Si}-\mathrm{CH}_{3}\right) .{ }^{13} \mathrm{C} \mathrm{NMR}$ $\left(\mathrm{CDCl}_{3}\right): \delta 166.0(\mathrm{C}=\mathrm{O}), 149.7$ (quaternary C), $148.9(\mathrm{Ar}-\mathrm{CH}), 146.7(\mathrm{Ar}-\mathrm{CH}), 142.5$ $\left(\mathrm{CH}=\mathrm{CHCO}_{2} \mathrm{Et}\right), 135.4$ (quaternary $\left.\mathrm{C}\right), 128.3(\mathrm{Ar}-\mathrm{CH}), 120.5\left(\mathrm{CH}=\mathrm{CHCO}_{2} \mathrm{Et}\right), 60.5$ $\left(\mathrm{CH}_{2} \mathrm{CH}_{3}\right), 14.1\left(\mathrm{CH}_{2} \mathrm{CH}_{3}\right),-0.76\left(\mathrm{Si}\left(\mathrm{CH}_{3}\right)_{2}\right) .{ }^{29} \mathrm{Si} \mathrm{NMR}\left(\mathrm{CDCl}_{3}\right): \delta$-2.88. Anal. Calc. for $\mathrm{C}_{13} \mathrm{H}_{19} \mathrm{NO}_{2} \mathrm{Si}$ : C 62.61, H 7.68, N 5.62, Si 11.26. Found: C 62.70, H 7.58, N 5.58, Si 11.34.

3-(4-G1-pyridin-3-yl)-acrylic acid ethyl ester 16: 3-bromo-4-G1-pyridine 13 (200 $\mathrm{mg}, 0.06 \mathrm{mmol})$ in $10 \mathrm{~mL}$ of DMF, $\mathrm{Pd}(\mathrm{OAc})_{2}(4.0 \mathrm{mg}, 12 \times 2.5 \mathrm{~mol} \%), \mathrm{PPh}_{3}(9.3 \mathrm{mg}$, $12 \times 5 \mathrm{~mol} \%), \mathrm{NEt}_{3}(0.5 \mathrm{~mL}, 3.5 \mathrm{mmol})$ and ethyl acrylate $(0.12 \mathrm{~mL}, 1.06 \mathrm{mmol})$. The crude material (ca. $0.4 \mathrm{~g}$ ) was diluted in $10 \mathrm{~mL}$ of technical methanol and placed into a membrane bag. This bag was then placed into a beaker containing $200 \mathrm{~mL}$ of methanol and the solution was slowly stirred overnight at room temperature. Evaporation of the internal solution of the bag afforded the 3-(4-G1-pyridin-3-yl)acrylic acid ethyl ester 16 as brown oil (171 mg, 80\% yield). ${ }^{1} \mathrm{H}$ NMR $\left(\mathrm{CDCl}_{3}\right)$ : $\delta$ 8.73 (br s, $12 \mathrm{H}, \mathrm{Ar}-\mathrm{H}), 8.45$ (br s, $12 \mathrm{H}, \mathrm{Ar}-\mathrm{H}), 7.91$ (d, $12 \mathrm{H},{ }^{3} \mathrm{~J}_{\mathrm{H}-\mathrm{H}}=16.2 \mathrm{~Hz}$, $\left.\mathrm{CH}=\mathrm{CHCO}_{2} \mathrm{Et}\right), 7.30($ br s, $12 \mathrm{H}, \mathrm{Ar}-\mathrm{H}), 6.34\left(\mathrm{~d}, 12 \mathrm{H},{ }^{3} \mathrm{~J}_{\mathrm{H}-\mathrm{H}}=16.2 \mathrm{~Hz}\right.$, $\left.\mathrm{CH}=\mathrm{CHCO}_{2} \mathrm{Et}\right), 4.25-4.15\left(\mathrm{~m}, 24 \mathrm{H}, \mathrm{CH}_{2} \mathrm{CH}_{3}\right), 1.40-1.05\left(\mathrm{~m}, 76 \mathrm{H}, \mathrm{CH}_{2} \mathrm{CH}_{3}, \mathrm{C}_{-} \mathrm{CH}_{2}-\right.$ C), 0.95-0.70 (br m, $24 \mathrm{H}, \mathrm{CH}_{2}-\mathrm{Si}-\mathrm{Ar}$ ), 0.48 (br s, $32 \mathrm{H}, \mathrm{CH}_{2}-\mathrm{Si}_{-} \mathrm{CH}_{2}$ ), 0.29 (s, $72 \mathrm{H}$, $\left.\mathrm{Si}\left(\mathrm{CH}_{3}\right)_{2}(\mathrm{Ar})\right) .{ }^{13} \mathrm{C} \mathrm{NMR}\left(\mathrm{CDCl}_{3}\right): \delta 166.1(\mathrm{C}=\mathrm{O}), 150.0$ (quaternary $\left.\mathrm{C}\right), 148.9,146.9$ (2 x Ar-CH), $142.6\left(\mathrm{CH}=\mathrm{CHCO}_{2} \mathrm{Et}\right), 135.5$ (quaternary $\left.\mathrm{C}\right), 128.5(\mathrm{Ar}-\mathrm{CH}), 120.6$ $\left(\mathrm{CH}=\mathrm{CHCO}_{2} \mathrm{Et}\right), 60.6\left(\mathrm{CH}_{2} \mathrm{CH}_{3}\right), 20.4,20.2,18.4,17.9,17.1\left(\mathrm{CH}_{2}\right), 14.2\left(\mathrm{CH}_{2} \mathrm{CH}_{3}\right)$, $-2.2\left(\mathrm{Si}\left(\mathrm{CH}_{3}\right)_{2}(\mathrm{Ar})\right) .{ }^{29} \mathrm{Si} \mathrm{NMR}\left(\mathrm{CDCl}_{3}\right): \delta 0.8$ (Si core), -2.5 (Si-Ar), -3.8 (Si inner). 
General procedure for the Sonogashira coupling reaction: ${ }^{(v)}$ To a solution of silylated pyridine ( 1 equiv) in dry tetrahydrofuran $(5 \mathrm{~mL}$ per mmol) were added $\mathrm{PdCl}_{2}\left(\mathrm{PPh}_{3}\right)_{2}(5 \mathrm{~mol} \%), \mathrm{PPh}_{3}(10 \mathrm{~mol} \%), \mathrm{NEt}_{3}$ (2 equiv), phenylacetylene (1.5 equiv) and copper iodide $(10 \mathrm{~mol} \%)$. The reaction mixture was then heated at reflux 16 hours. The mixture was cooled down to room temperature, diluted with water, extracted with ethyl acetate and dried over $\mathrm{MgSO}_{4}$. The resulting solution was filtered through a short plug of Celite and evaporated to dryness. The crude material was purified by column chromatography using an appropriate mixture of ethyl acetate and hexane or by passive dialysis.

3-phenylethynyl-4-trimethylsilanylpyridine 5: 3-bromo-4-trimethylsilanylpyridine $2(0.30 \mathrm{~g}, 1.3 \mathrm{mmol})$ in $10 \mathrm{~mL}$ of THF, $\mathrm{PdCl}_{2}\left(\mathrm{PPh}_{3}\right)_{2}(45.8 \mathrm{mg}, 5 \mathrm{~mol} \%), \mathrm{PPh}_{3}(34.2$ $\mathrm{mg}, 10 \mathrm{~mol} \%), \mathrm{NEt}_{3}(0.4 \mathrm{~mL}, 2.6 \mathrm{mmol})$, phenylacetylene $(0.21 \mathrm{~mL}, 1.95 \mathrm{mmol})$ and copper iodide (24.8 mg, $10 \mathrm{~mol} \%)$. Column chromatography using ethyl acetate/hexane (1/5) afforded the 3-phenylethynyl-4-trimethylsilanylpyridine $\mathbf{5}$ as colorless oil (0.29 g, 88\% yield). ${ }^{1} \mathrm{H}$ NMR $\left(\mathrm{CDCl}_{3}\right): \delta 8.72$ (br s, $\left.1 \mathrm{H}, \mathrm{Ar}-\mathrm{H}\right), 8.48(\mathrm{~d}$, $\left.1 \mathrm{H},{ }^{3} \mathrm{~J}_{\mathrm{H}-\mathrm{H}}=4.7 \mathrm{~Hz}, \mathrm{Ar}-\mathrm{H}\right), 7.58-7.50(\mathrm{~m}, 2 \mathrm{H}, 2$ x Ar-H), 7.40-7.34 (m, $4 \mathrm{H}, 4$ x Ar$\mathrm{H}), 0.44\left(\mathrm{~s}, 9 \mathrm{H}, \mathrm{Si}-\mathrm{CH}_{3}\right) .{ }^{13} \mathrm{C} \mathrm{NMR}\left(\mathrm{CDCl}_{3}\right): \delta 152,1$ (Ar-CH), 151.5 (quaternary C), 147.3 (Ar-CH), 131.3 (Ar-CH), 128.7, 128.5 (2 x Ar-CH), 127.9 (Ar-CH), 125.4 (quaternary C), 122.7 (quaternary $\mathrm{C}), 94.8(\mathrm{C} \equiv C \mathrm{Ph}), 87.9(\mathrm{C} \equiv \mathrm{CPh}),-1.7\left(\mathrm{Si}\left(\mathrm{CH}_{3}\right)_{2}\right)$. ${ }^{29} \mathrm{Si} \mathrm{NMR}\left(\mathrm{CDCl}_{3}\right): \delta$-3.13. Anal. Calc. for $\mathrm{C}_{16} \mathrm{H}_{17} \mathrm{NSi}$ : C 76.44, H 6.82, N 5.57, Si 11.17. Found: C 76.35, H 6.76, N 5.48, Si 11.23.

[3-phenylethynyl-4-G1-pyridine] 17: 3-bromo-4-G1-pyridine 13 (200 mg, 0.06 mmol) in $10 \mathrm{~mL}$ of THF, $\mathrm{PdCl}_{2}\left(\mathrm{PPh}_{3}\right)_{2}(24.8 \mathrm{mg}, 12 \times 5 \mathrm{~mol} \%), \mathrm{PPh}_{3}(18.5 \mathrm{mg}, 12 \times 10$ mol\%), $\mathrm{NEt}_{3}(0.2 \mathrm{~mL}, 1.4 \mathrm{mmol})$, phenylacetylene $(0.12 \mathrm{~mL}, 1.06 \mathrm{mmol})$ and copper 
iodide (13.5 mg, 12x10 mol\%). The crude material (ca. $0.3 \mathrm{~g}$ ) was diluted in $10 \mathrm{~mL}$ of technical dichloromethane and placed into a membrane bag. This bag was then placed into a beaker containing $200 \mathrm{~mL}$ of dichloromethane and the solution was slowly stirred overnight at room temperature. Evaporation of the internal solution of the bag afforded the 3-phenylethynyl-4-G1-pyridine 17 as brown oil (149 mg, 70\% yield). ${ }^{1} \mathrm{H}$ NMR $\left(\mathrm{CDCl}_{3}\right): \delta 8.63$ (br s, $\left.12 \mathrm{H}, \mathrm{Ar}-\mathrm{H}\right), 8.47$ (br s, $\left.12 \mathrm{H}, \mathrm{Ar}-\mathrm{CH}\right), 7.90-7.60$ (br m, 12 H, Ar-H), 7.54-7.46 (m, 12 H, Ar-H), 7.40-7.16 (br m, 48 H, Ar-H), 1.50-1.15 (br m, $40 \mathrm{H}, \mathrm{C}-\mathrm{CH}_{2}-\mathrm{C}$ ), 1.05-0.90 (br m, $24 \mathrm{H}, \mathrm{CH}_{2}-\mathrm{Si}-\mathrm{Ar}$ ), 0.70-0.35 (br m, $32 \mathrm{H}, \mathrm{CH}_{2}-$ $\left.\mathrm{Si}-\mathrm{CH}_{2}\right), 0.33\left(\mathrm{~s}, 72 \mathrm{H}, \mathrm{Si}-\mathrm{CH}_{3}\right),{ }^{13} \mathrm{C} \mathrm{NMR}\left(\mathrm{CDCl}_{3}\right): \delta 151.2(\mathrm{Ar}-\mathrm{CH}), 150.3$ (quaternary C), 146.9, 132.0, 129.6, 128.5, 128.4 (Ar-CH), 121.5 (quaternary C), 93.8 $(\mathrm{C} \equiv C \mathrm{Ph}), 88.1(\mathrm{C} \equiv \mathrm{CPh}), 21.0,19.3,18.5,17.9,17.2\left(\mathrm{CH}_{2}\right),-2.8\left(\mathrm{Si}\left(\mathrm{CH}_{3}\right)_{2}\right) .{ }^{29} \mathrm{Si}$ NMR $\left(\mathrm{CDCl}_{3}\right): \delta 0.7$ (Si core), -3.2 (Si-Ar), -3.9 (Si inner).

\section{General procedure for the desilylation reaction using tetrabutylammonium}

fluoride: ${ }^{(v i)}$ To a solution of silylated pyridine (1 equiv) in tetrahydrofuran $(5 \mathrm{~mL}$ per mmol) was added a solution of tetrabutylammonium fluoride (2 equiv, $1 \mathrm{M}$ in solution in THF) at room temperature. The reaction mixture was stirred overnight at room temperature. The mixture was extracted with ethyl acetate and a solution of saturated $\mathrm{NaHCO}_{3}$ solution, washed of the organic layers with water, dried over $\mathrm{MgSO}_{4}$ and evaporated to dryness. The crude was then filtered through a short plug of silica using ethyl acetate as eluent.

3-p-tolylpyridine 6: ${ }^{(v i i)} 3$-p-tolyl-4-trimethysilanylpyridine 3 (300 mg, $1.2 \mathrm{mmol}$ ) in 6 $\mathrm{mL}$ of THF, and $\mathrm{nBu}_{4} \mathrm{NF}(2.5 \mathrm{~mL}, 1 \mathrm{M}$ solution in THF). Filtration through a plug of silica using ethyl acetate as eluent afforded the 3-p-tolylpyridine 6 as white powder (198 mg, 94\%). 
G1-(3-p-tolylPy $)_{12} 15(250 \mathrm{mg}, 0.07 \mathrm{mmol})$ in $5 \mathrm{~mL}$ of THF, and $\mathrm{nBu}_{4} \mathrm{NF}(1.7 \mathrm{~mL}, 1$ M solution in THF). Filtration through a plug of silica using ethyl acetate as eluent afforded the 3-p-tolylpyridine 6 as white powder (130 mg, 90\%). ${ }^{1} \mathrm{H} \mathrm{NMR}\left(\mathrm{CDCl}_{3}\right): \delta$ $8.75\left(\mathrm{~d}, 1 \mathrm{H},{ }^{3} \mathrm{~J}_{\mathrm{H}-\mathrm{H}}=2.7 \mathrm{~Hz}, \mathrm{Ar}-\mathrm{H}\right), 8.47\left(\mathrm{dd}, 1 \mathrm{H},{ }^{3} \mathrm{~J}_{\mathrm{H}-\mathrm{H}}=4.8 \mathrm{~Hz},{ }^{4} \mathrm{~J}_{\mathrm{H}-\mathrm{H}}=0.9 \mathrm{~Hz}, \mathrm{Ar}-\right.$ $\mathrm{H}), 7.72\left(\mathrm{dm}, 1 \mathrm{H},{ }^{3} \mathrm{~J}_{\mathrm{H}-\mathrm{H}}=8.1 \mathrm{~Hz}\right), 7.37\left(\mathrm{~d}(\mathrm{AB}), 2 \mathrm{H},{ }^{3} \mathrm{~J}_{\mathrm{H}-\mathrm{H}}=8.4 \mathrm{~Hz}\right.$, Ar-H Phenyl), 7.26-7.18 (m, $1 \mathrm{H}$, Ar-H), $7.17\left(\mathrm{~d}(\mathrm{AB}), 2 \mathrm{H},{ }^{3} \mathrm{~J}_{\mathrm{H}-\mathrm{H}}=8.4 \mathrm{~Hz}\right.$, Ar-H Phenyl), 2.30 (s, $\left.3 \mathrm{H}, \mathrm{Ar}-\mathrm{CH}_{3}\right) .{ }^{13} \mathrm{C} \mathrm{NMR}\left(\mathrm{CDCl}_{3}\right): \delta 148.0(\mathrm{Ar}-\mathrm{CH}), 138.0,136.6,134.8(3 \mathrm{x}$ quaternary C), 134.1, 129.7, 128.1, 126.9, $123.5(\mathrm{Ar}-\mathrm{CH}), 21.4\left(\mathrm{Ar}-\mathrm{CH}_{3}\right)$. Anal. Calc. for $\mathrm{C}_{12} \mathrm{H}_{11} \mathrm{~N}$ : C 85.17, H 6.55, N 8.28. Found: C 85.10, H 6.48, N 8.16.

3-pyridin-3-yl-acrylic acid ethyl ester 7: ${ }^{\text {(viii) } 3-(4-t r i m e t h y l s i l a n y l p y r i d i n-3-y l)-~}$ acrylic acid ethyl ester 4 (240 mg, $0.96 \mathrm{mmol})$ in $5 \mathrm{~mL}$ of THF, and $\mathrm{nBu} 4 \mathrm{NF}(1.9 \mathrm{~mL}$, $1 \mathrm{M}$ solution in THF). Filtration through a plug of silica using ethyl acetate as eluent afforded the 3-pyridin-3-yl-acrylic acid ethyl ester 7 as white powder (155 mg, 91\%). 3-(4-G1-pyridin-3-yl)-acrylic acid ethyl ester 16 (200 mg, $0.055 \mathrm{mmol})$ in $5 \mathrm{~mL}$ of THF, and $\mathrm{nBu}_{4} \mathrm{NF}(1.3 \mathrm{~mL}, 1 \mathrm{M}$ solution in THF). Filtration through a plug of silica using ethyl acetate as eluent afforded the 3-pyridin-3-yl-acrylic acid ethyl ester 7 as white powder $(101 \mathrm{mg}, 85 \%) .{ }^{1} \mathrm{H} \mathrm{NMR}\left(\mathrm{CDCl}_{3}\right): \delta 8.70(\mathrm{~s}, 1 \mathrm{H}, \mathrm{Ar}-\mathrm{H}), 8.55(\mathrm{~d}, 1 \mathrm{H}$, $\left.{ }^{3} \mathrm{~J}_{\mathrm{H}-\mathrm{H}}=4.7 \mathrm{~Hz}, \mathrm{Ar}-\mathrm{H}\right), 7.79\left(\mathrm{dm}, 1 \mathrm{H},{ }^{3} \mathrm{~J}_{\mathrm{H}-\mathrm{H}}=8.0 \mathrm{~Hz}\right), 7.62\left(\mathrm{~d}, 1 \mathrm{H},{ }^{3} \mathrm{~J}_{\mathrm{H}-\mathrm{H}}=16.2 \mathrm{~Hz}\right.$, $\left.\mathrm{CH}=\mathrm{CHCO}_{2} \mathrm{Et}\right), 7.30-7.26(\mathrm{~m}, 1 \mathrm{H}, \mathrm{Ar}-\mathrm{H}), 6.46\left(\mathrm{~d}, 1 \mathrm{H},{ }^{3} \mathrm{~J}_{\mathrm{H}-\mathrm{H}}=16.2 \mathrm{~Hz}\right.$, $\left.\mathrm{CH}=\mathrm{CHCO}_{2} \mathrm{Et}\right), 4.23\left(\mathrm{q}, 2 \mathrm{H},{ }^{3} \mathrm{~J}_{\mathrm{H}-\mathrm{H}}=6.0 \mathrm{~Hz}, \mathrm{CH}_{2} \mathrm{CH}_{3}\right), 1.29\left(\mathrm{t}, 3 \mathrm{H},{ }^{3} \mathrm{~J}_{\mathrm{H}-\mathrm{H}}=6.0 \mathrm{~Hz}\right.$, $\left.\mathrm{CH}_{2} \mathrm{CH}_{3}\right) .{ }^{13} \mathrm{C}$ NMR $\left(\mathrm{CDCl}_{3}\right): \delta 166.1(\mathrm{C}=\mathrm{O}), 150.8,149.5(\mathrm{Ar}-\mathrm{CH}), 140.7$ $\left(\mathrm{CH}=\mathrm{CHCO}_{2} \mathrm{Et}\right), 134.1,123.6(\mathrm{Ar}-\mathrm{CH}), 120.4\left(\mathrm{CH}=\mathrm{CHCO}_{2} \mathrm{Et}\right), 112.1$ (quaternary $\left.\mathrm{C}\right)$, 60.6 $\left(\mathrm{CH}_{2} \mathrm{CH}_{3}\right), 14.1\left(\mathrm{CH}_{2} \mathrm{CH}_{3}\right)$. Anal. Calc. for $\mathrm{C}_{10} \mathrm{H}_{11} \mathrm{~N}$ : C 67.78, H 6.26, N 7.90. Found: C 67.63, H 6.34, N 7.95. 
3-phenylethynylpyridine 8: ${ }^{(i x)}$ 3-phenylethynyl-4-trimethysilanylpyridine 5 (180 mg, $0.72 \mathrm{mmol})$ in $5 \mathrm{~mL}$ of $\mathrm{THF}$, and $\mathrm{nBu}_{4} \mathrm{NF}(1.4 \mathrm{~mL}, 1 \mathrm{M}$ solution in THF). Filtration through a plug of silica using ethyl acetate as eluent afforded the 3phenylethynylpyridine 8 as white solid (122 mg, 93\%).

3-phenylethynyl-4-G1-pyridine $17(200 \mathrm{mg}, 0.054 \mathrm{mmol})$ in $5 \mathrm{~mL}$ of THF, and $\mathrm{nBu}_{4} \mathrm{NF}(1.3 \mathrm{~mL}, 1 \mathrm{M}$ solution in THF). Filtration through a plug of silica using ethyl acetate as eluent afforded the 3-phenylethynylpyridine $\mathbf{8}$ as slight orange solid (95 mg, 81\%). ${ }^{1} \mathrm{H}$ NMR $\left(\mathrm{CDCl}_{3}\right): \delta 8.77(\mathrm{~s}, 1 \mathrm{H}, \mathrm{Ar}-\mathrm{H}), 8.53\left(\mathrm{~d}, 1 \mathrm{H},{ }^{3} \mathrm{~J}_{\mathrm{H}-\mathrm{H}}=4.7 \mathrm{~Hz}, \mathrm{Ar}-\right.$ H), $7.80\left(\mathrm{dm}, 1 \mathrm{H},{ }^{3} \mathrm{~J}_{\mathrm{H}-\mathrm{H}}=8.0 \mathrm{~Hz}\right), 7.56-7.52(\mathrm{~m}, 2 \mathrm{H}, \mathrm{Ar}-\mathrm{CH})$, 7.37-7.34 (m, $3 \mathrm{H}, \mathrm{Ar}-$ $\mathrm{H})$, 7.29-7.24 (m, $1 \mathrm{H}, \mathrm{Ar}-\mathrm{CH}) .{ }^{13} \mathrm{C} \mathrm{NMR}\left(\mathrm{CDCl}_{3}\right): \delta 152.0,149.3,138.6,131.7$, 128.8, 128.4, $127.9(\mathrm{Ar}-\mathrm{CH}), 126.3,123.1$ (quaternary $\mathrm{C}), 93.9(\mathrm{C} \equiv C \mathrm{Ph}), 88.7$

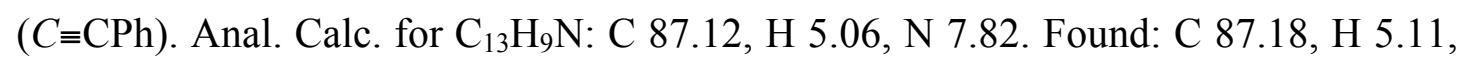
N 7.68.

\section{$\underline{\text { References: }}$}

(i) (a) Gribble, G. W.; Saulnier, M. G. Tetrahedron Lett. 1980, 21, 4137-4140. (b) Gribble, G. W.; Saulnier, M. G. Heterocycles 1993, 35, 151-169. (c) Comins, D. L.; Myoung, Y. C. J. Org. Chem. 1990, 55, 292-298.

(ii) Effenberger, F.; Daub, W. Chem. Ber. 1991, 124, 2119-2125.

(iii) (a) Karig, G.; Spencer, J. A.; Gallagher, T. Org. Lett. 2001, 3, 835-838. (b) Miyaura, N.; Suzuki, A. Chem. Rev. 1995, 95, 2457-2483.

(iv) (a) Anson, M. S.; Mirza, A. R.; Tonks, L.; Williams, J. M. J. Tetrahedron Lett. 1999, 40, 7147-7150. (b) Whitcombe, N. J.; Hii, K. K.; Gibson, S. E. Tetrahedron 2001, 57, 7449-7476. (c) Beletskaya, I. P.; Cheprakov, A. V. Chem. Rev. 2000, 100, 3009-3066. 
(v) (a) Sonogashira, K. in Metal-Catalyzed Cross-Coupling Reactions; F. Diederich, P. J. Stang, Eds.; Wiley-VCH: New York, 1998. Sonogashira, K. J. Organomet. Chem. 2002, 653, 46-49. (b) Louerat, F.; Gros, P.; Fort, Y. Tetrahedron Lett. 2003, 44, 5613-5616.

(vi) (a) Boehm, T. L.; Showalter, H. D. H. J. Org. Chem. 1996, 61, 6498-6499. (b) Fensterbank, L.; Malacria, M.; Siebarth, S. McN. Synthesis 1997, 817-854. (c) Kumada, M.; Tamao, K.; Yoshida, J. J. Organomet. Chem. 1982, 239, 115-132.

(vii) (a) Inada, K.; Miyaura, N. A. Tetrahedron 2000, 56, 8661-8664. (b) Benniston, A. C. Tetrahedron Lett. 1997, 38, 8279-8282. (c) Ishikura, M.; Kamada, M.; Terashima, M. Heterocycles 1984, 22, 265-268.

(viii) Draper, T. L.; Thomas, R. Synlett 1995, 2, 157-158.

(ix) (a) Appukkuttan, P.; Dehaen, W.; Van der Eycken, E. Eur. J. Org. Chem. 2003, 4713-4716. (b) Soheili, A.; Albaneze-Walker, J.; Murry, J. A.; Dormer, P. G.; Hughes, D. L. Org. Lett. 2003, 5, 4191-4194. (c) Ishikura, M.; Kamada, M.; Ohta, T.; Terashima, M. Heterocycles 1984, 22, 2475-2478. (d) Castro, C. E.; Gaughan, E. J.; Owsley, D. C. J. Org. Chem. 1966, 31, 4071-4078. 
Figure 1: ${ }^{1} \mathrm{H}$ NMR of the crude mixture of compound 12.

(8)

$\xi$
$\xi$
$\Xi$

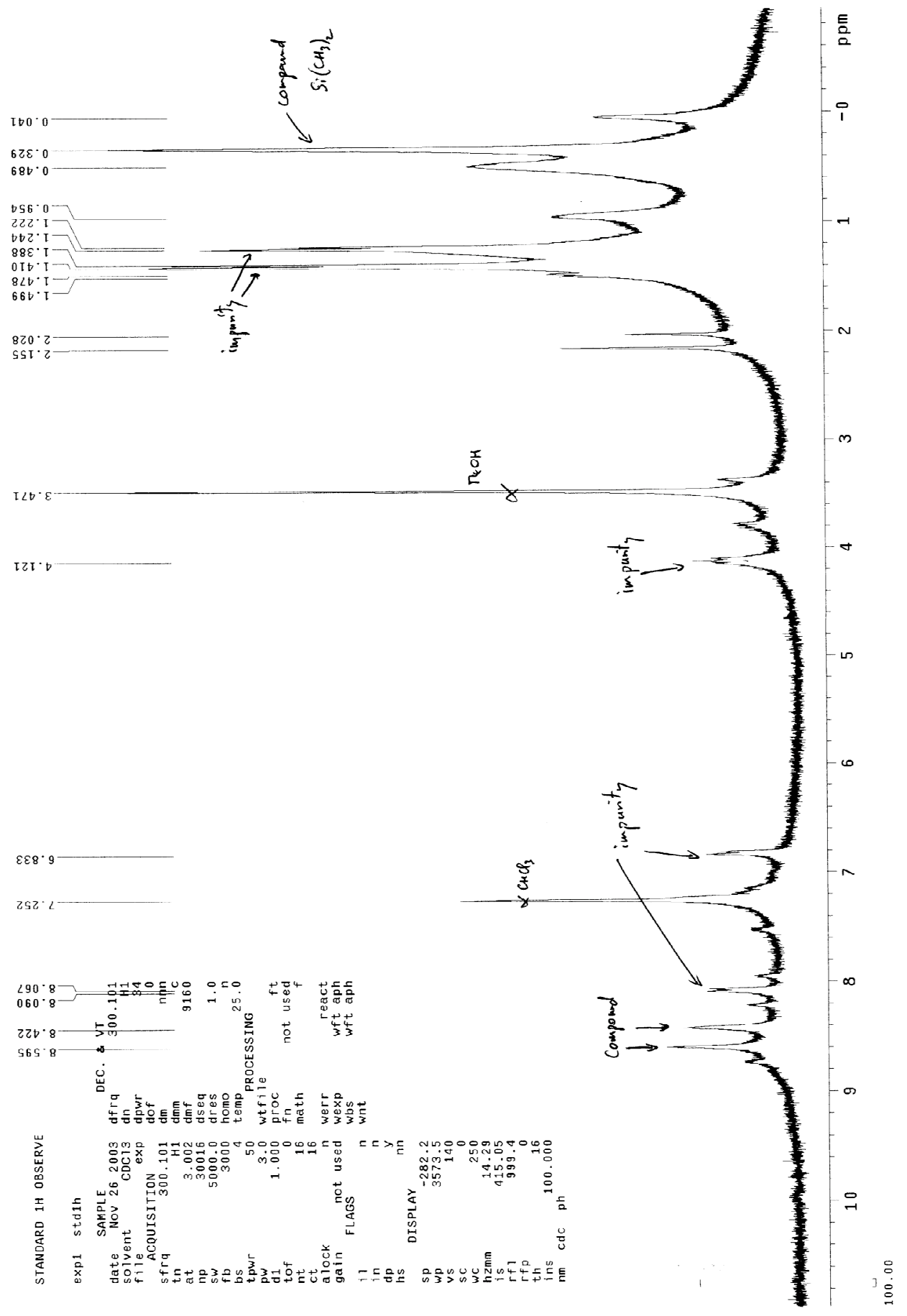


Figure 2: ${ }^{1} \mathrm{H}$ NMR of the filtrate from dialysis.
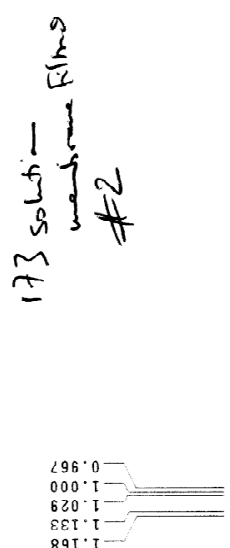

>.

102

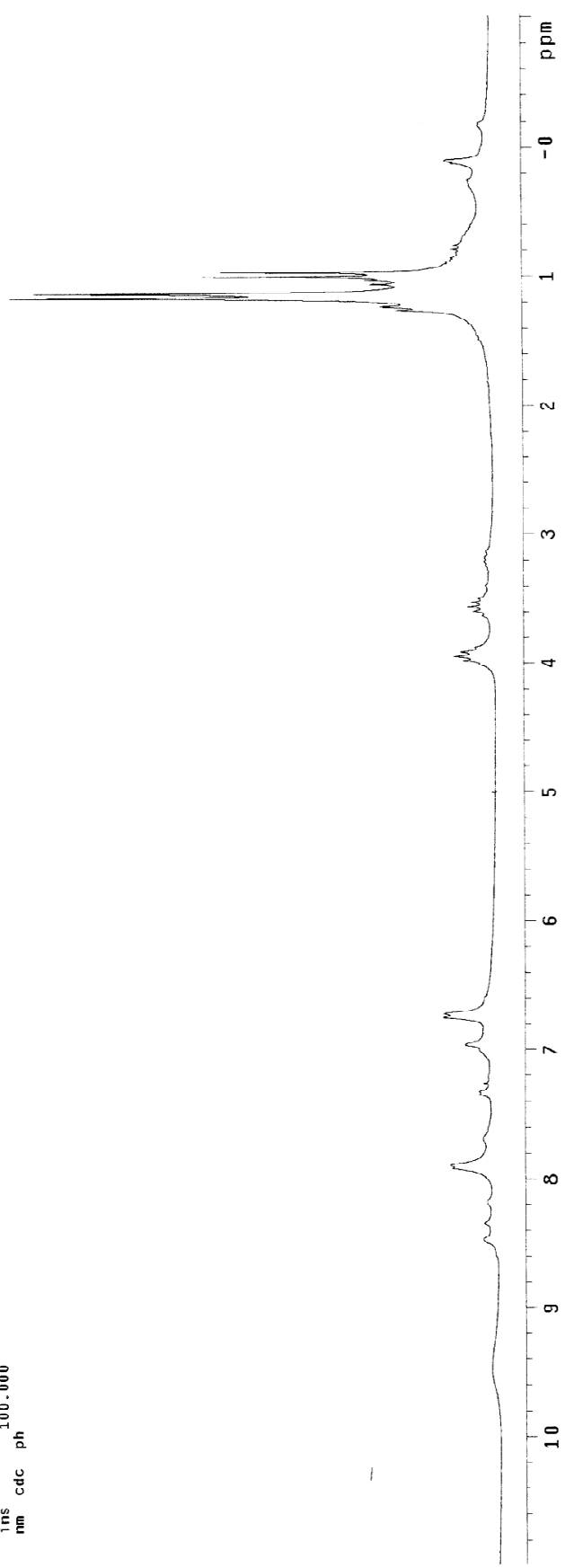


Figure 3: ${ }^{1} \mathrm{H}$ NMR of the dialyzed compound 12.
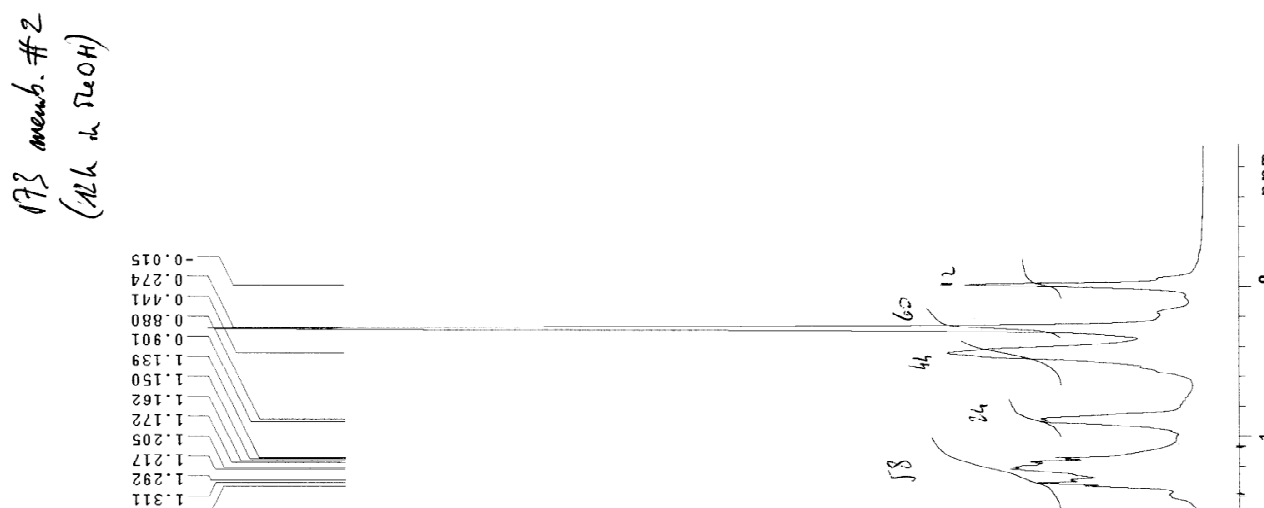

${ }_{\varepsilon \varepsilon \varepsilon^{\top} \mathrm{I}}^{\mathrm{I}}$ -

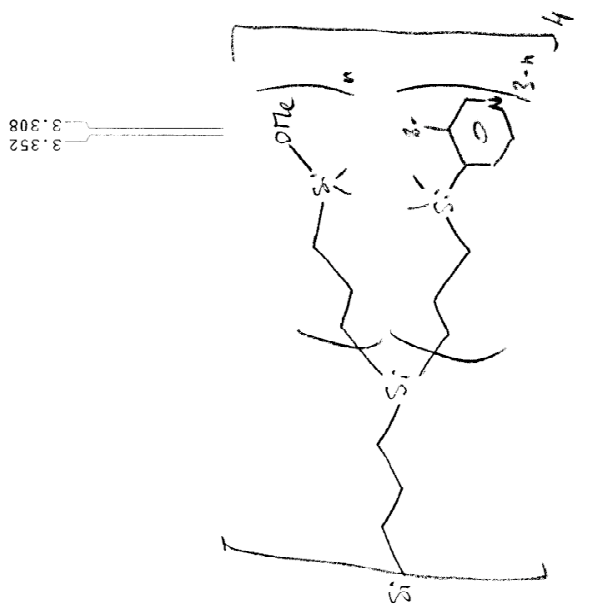

โ $8 I^{\circ}$
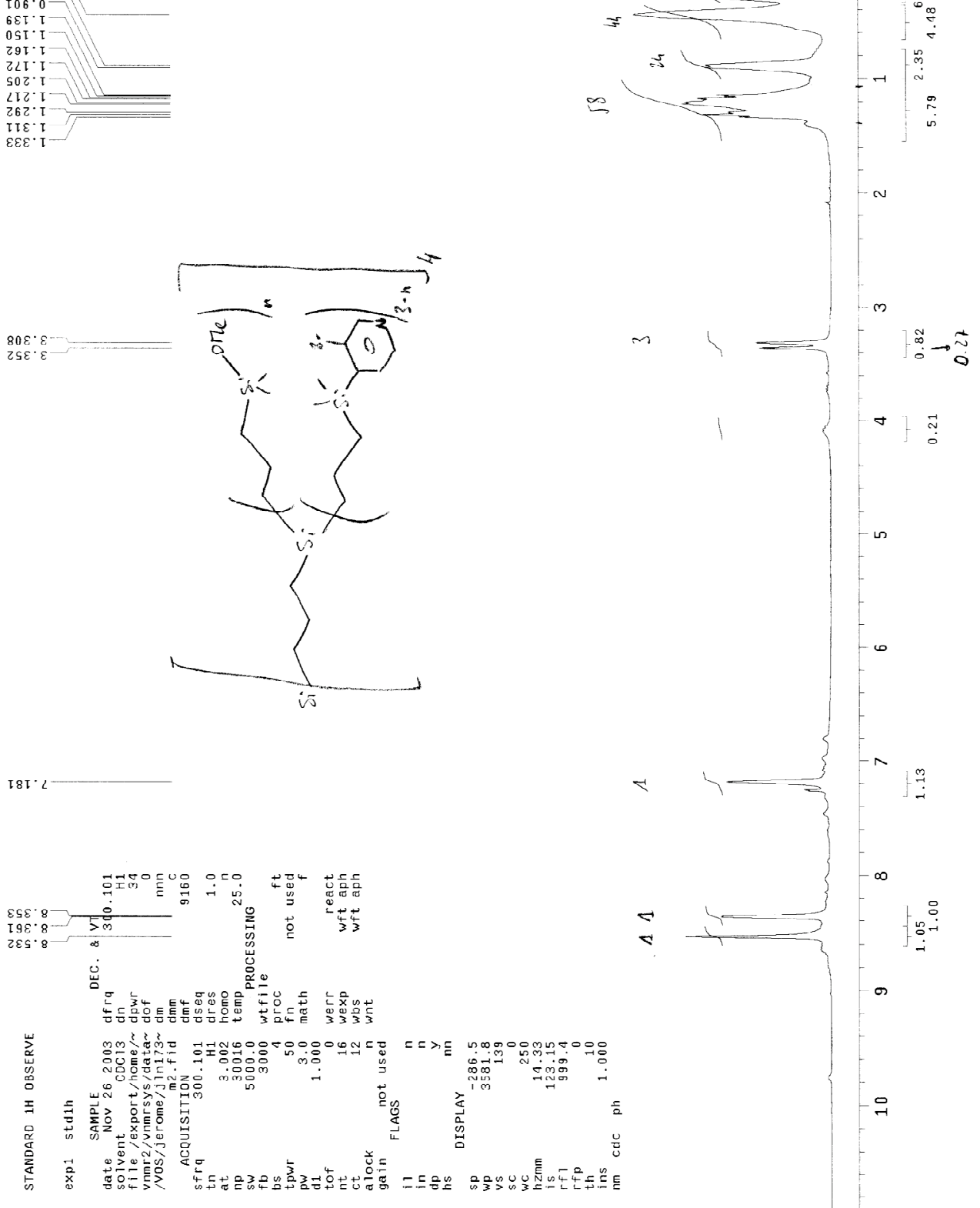
Figure $4:{ }^{13} \mathrm{C}$ NMR of the dialyzed compound 12 .

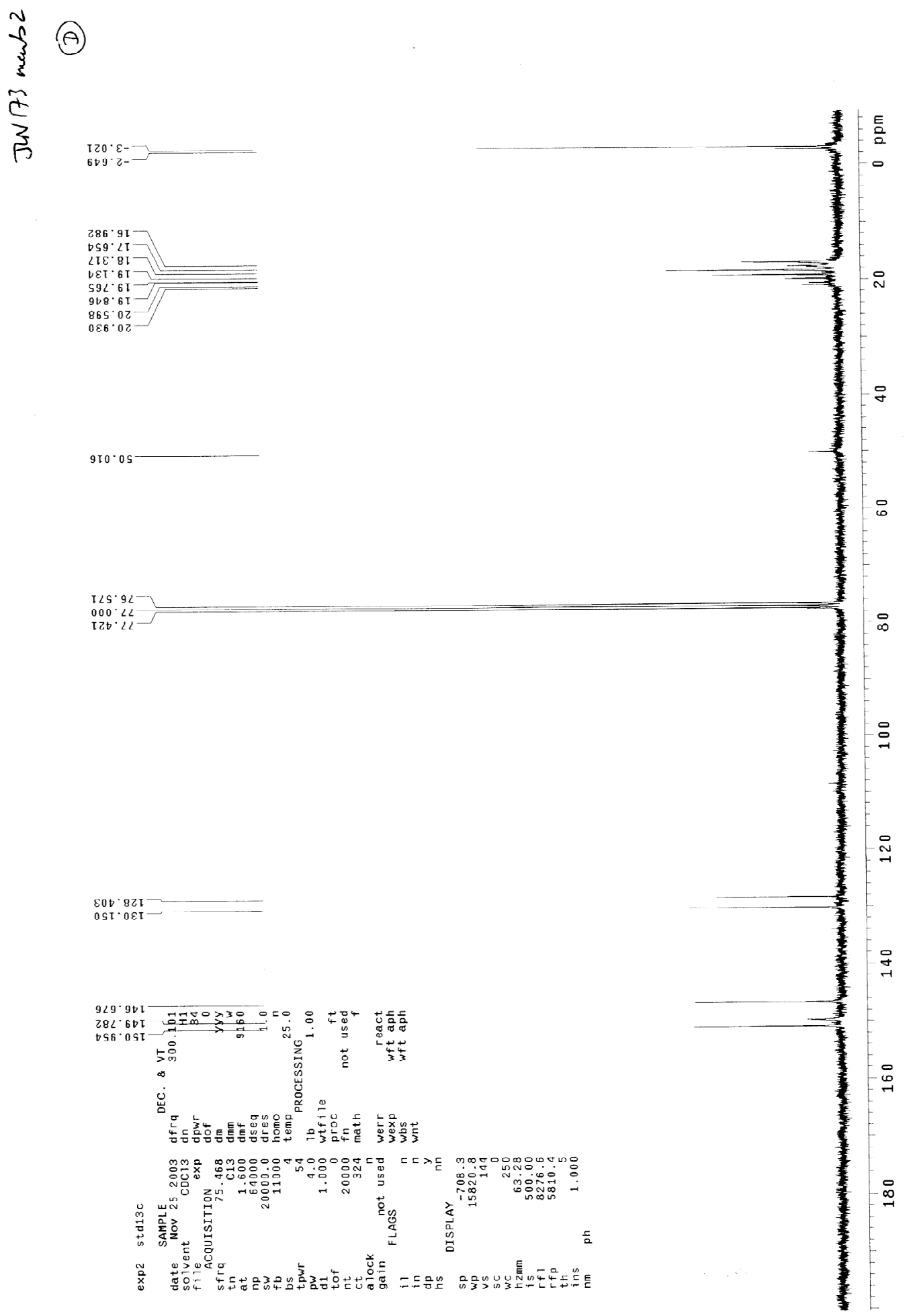

